\title{
Stem-cell replication to treat blood diseases
}

$\mathrm{H}$ uman clinical trials are set to start in December to test an innovative approach to treating life-threatening blood diseases, such as leukemia.

A team of researchers from the Institute for Research in Immunology and Cancer (IRIC) at the Université de Montréal have discovered a new molecule, UM171, that can be used to increase the number of stem cells found in umbilical cord blood. The innovative finding, which has been described as a world breakthrough, was published Sept. 19, 2014, in Science.

According to the team's principal investigator, Dr. Guy Sauvageau, this new synthetic molecule could "multiply by 10 the number of units of cord blood available for transplantation in humans" and would also substantially reduce complications associated with stem cell transplant.

When no compatible donor can be found, stem cells from umbilical cords are transplanted to treat blood diseases such as leukemia, myeloma and lymphoma. However, the number of stem cells obtained from a unit of blood from an umbilical cord is usually too low to treat an adult. In addition, finding a source of stem cells for smaller ethnic groups can be difficult. With the new UM171 molecule, it would be possible, according to researchers, to harvest stem cells in culture, and produce enough to treat adults.

"It was a bit of a fluke," says Anne Marinier, coauthor of the paper and a chemist who practically hand-picked the synthetic molecule out of a library of 125000 compounds stored at the institute. "When we saw how reactive it was on blood stem cells, we were extremely surprised and delighted,"

Centre de recherche Hôpital Maisonneuve-Rosemont, who will lead the human clinical trial, this new approach could potentially increase accessibility to treatment by $30 \%$ to $40 \%$.

The blood of a newborn's umbilical cord is a rich source of hematopoietic stem cells for transplantation. Because the donor newborn's immune system is still immature, the stem cells are less likely to induce an adverse immune reaction in the receiver. Moreover, it is not necessary that the immunological compatibility between donor and recipient is perfect, unlike a bone marrow transplant.

says Marinier, who is IRIC's director of medicinal chemistry.

Tests on mice, then monkeys, showed the potent effect of UM171 in promoting the proliferation of blood stem cells. The molecule is now being patented for potential commercialization. Marinier credits her experience as a chemist at a pharmaceutical company with helping her choose the right compound. She says it's the first time she's seen that her research "could make a difference in someone's life and prevent them from dying. It is extremely rewarding for the team and the institute."

Producing the stem cells involves using a bioreactor at a University of Toronto bioengineering lab. The bioreactor prevents stem cell differentiation and offers a medium in which they can proliferate without risking contamination. In short, the bioreactor preserves the integrity of the stem cells, says Peter Zandstra, a bioengineering researcher and director of the lab that houses the bioreactor. "We add small amounts of media, and we do it in a way that is cost-effective and reactive. There is a real clinical need here."

According to Dr. Jean Roy, of the
Cord blood transplants have been used worldwide at an increasing rate since the late $1990 \mathrm{~s}$, but only $5 \%$ of adults can be treated efficiently this way, according to Roy. That could change. "It could become a life-saving option, especially for non-Caucasian patients who have difficulties finding compatible donors," believes Roy.

In the first and second phase of the human clinical trial, the Centre of Excellence for Cellular Therapy at Hôpital Maisonneuve-Rosemont will be distributing the grafts obtained from UM171 to 15 patients in Québec City, Montréal and Vancouver. The first results should be available in December 2015 .

"The number of people waiting for a transplant in Canada is impossible to know," says Roy, "but there are about one thousand allografts annually. This new molecule could help increase the number of grafts by more than $30 \%$. And I am being conservative." The main beneficiaries will be nonwhite patients who do not have compatible donors. Veronique Morin, Montréal, Que.

CMAJ 2014. DOI:10.1503/cmaj.109-4925 